Bustos, E., Bozzano, H., Molina, A. (2016). La riqueza de un territorio que ha vivido los avatares de la violencia. Revista Lebret, 8. Bucaramanga, Colombia: Universidad Santo Tomás, pp. 77 - 90. ISSN: 2145-5996

\title{
La riqueza de un territorio que ha vivido los avatares de la violencia*
}

\section{The wealth of a territory that has lived the avatars of violence}

Edier Hernan Bustos Velazco ${ }^{I}$

Horacio Bozzano 2

Adela Molina Andrade ${ }^{3}$

\begin{abstract}
Resumen
El artículo presenta de manera suscinta la relación de los hitos, huellas e improntas (Bozzano, 2009) de la historia territorial del municipio de Quibdó (capital del departamento de Chocó), y su impacto en las concepciones de territorio de sus habitantes, lo que permite reflexionar en las implicaciones que tienen para el sujeto territorial aspectos como la minería, los cultivos ilícitos y la violencia, actos que han afectado su propia historia territorial y que subyace en sentimientos por el territorio vivido, transformado, sometido y abusado.
\end{abstract}

\section{Palabras clave}

Territorio, cultura, contexto, hitos.

\section{Códigos de clasificación JEL: D13, I31, J61, Q30. Q51}

\begin{abstract}
The article briefly describes the relation between the landmarks of the municipality of Quibdo (capital of the department of Chocó) and its impact on the conceptions of territory of its inhabitants, which allows reflecting on the implications for the territorial subject of aspects such as mining, illicit cultivation and violence, acts that have affected their own territorial history and which underlie feelings for the territory lived, transformed, subjected and abused.
\end{abstract}

\section{Keywords}

Territory, culture, context, milestones.

Artículo derivado del proyecto: Concepciones de territorio de docentes universitarios formadores de profesionales de las ciencias de la tierra (pct): estudio comparado en dos universidades públicas ubicadas en contextos culturalmente diferenciados. Financiado por la Universidad Distrital Francisco José de Caldas.

1 Estudiante Doctorado Interinstitucional en Educación (DIE) énfasis Enseñanza de las Ciencias Contexto y Diversidad Cultural. Estudiante Doctorado en Geografía FaHCE (UNLP). Docente Universidad Distrital Francisco José de Caldas. Correo electrónico: ehbustosv@udistrital.edu.co

2 Docteur en Géographie-Amenagement-Urbanisme. Docente investigador Universidad Nacional de La Plata. FaHCE (UNLP). (Argentina). Correo electrónico: hbozzano@fahce.unlp.edu.ar

3 Doctora en Educación, Área de Concentración Enseñanza de las Ciencias y las Matemáticas. Docente Universidad Distrital Francisco José de Caldas. Correo electrónico: amolina@udistrital.edu.co 


\section{Introducción}

El trabajo desarrollado por Mosquera (2012) se configura en el eje central de este documento; el autor presenta de manera precisa los acontecimientos que demarcaron la historia territorial del municipio de Quibdó, que articulado con otras investigaciones, noticias, narraciones y entrevistas a la comunidad (E., 2014) ${ }^{4}$ nos permite determinar su relación con las dimensiones de territorio definidas en Bustos (2015), en un departamento rico en todo tipo de biodiversidad pero olvidado por el Estado.

Por lo expuesto hasta aquí y basados en el trabajo de Bustos (2015), el presente trabajo plantea que las concepciones de territorio están determinadas por el origen y prácticas culturales. Así, el trabajo desarrollado por Molina et al., (2014) permite determinar que las concepciones no se transmiten sino que se construyen en el interior de los grupos. El individuo construye su conocimiento en entornos sociales y con prácticas culturales de modo que en el transcurso de tales prácticas no recibe un conocimiento ya elaborado, sino que este hace una elaboración del conocimiento por sí mismo a partir de experiencias obtenidas, tanto por su entorno cultural como por las interacciones interpersonales que constituyen las características de la cultura.

Para llegar a caracterizar las concepciones y su vínculo con los hitos, huellas e improntas, Bozzano (2009), se realizó una revisión histórica a partir de fuentes bibliográficas, comunicados de prensa y se indagó en las comunidades directamente a partir de una entrevista semiestructurada aplicada a personas oriundas del territorio, lo que permitió entrelazar dimensiones teóricas, historias territoriales y narrativas, para así determinar la concepción de territorio que tiene la comunidad.

\section{Organización territorial actual de Quibdó}

Se inicia por una ubicación en la actualidad en el contexto de la capital del departamento de Chocó, con numerosos acontecimientos e íconos en su historia: su poblamiento ancestral indígena, luego la presencia de comunidades negras, con prácticas culturales, lo que configura un contexto culturalmente diferenciado respecto al resto del país. Contexto que se ha visto afectado por múltiples acontecimientos, entre ellos, la extracción y explotación indiscriminada de sus recursos, y a partir de 1988 la violencia que arreció y cambió formas de vida de las comunidades, específicamente con la presencia de grupos paramilitares y guerrilla; y en la actualidad la irrupción del emprendimiento de megaminería. Aspectos que calan en las personas oriundas de la región, quienes manifiestan:

[...] todo el departamento este viviendo una época pos Uribe, hemos visto la llegada de muchas personas, con diferentes expectativas, trajo mucha gente de afuera, algunos con buenas intenciones otros con malas [...], si tú te desplazas

4 Forma para hacer referencia a los entrevistados en el municipio de Quibdó (Chocó). 
ves mucho minero, gente del interior, eso se hizo muy fuerte después de Uribe, eso es como una especie de invasión, después de que se ganó la guerra, lo que hay ahora es una conquista después de una guerra (E., 2014).

Al hablar de pos Uribe, hacen referencia al mandato del presidente Álvaro Uribe Vélez entre el 2002 y el 2010, período en que con la excusa de la llamada Seguridad Democrática en Colombia se cometieron abusos a la población civil, entre ellos, los llamados falsos positivos de las Fuerzas Armadas legalmente constituidas por el Estado, como es el caso del asesinato de líderes campesinos, entre ellos Marino López Mena; el caso de mayor resonancia en el país asociado a las actividades extralegales del general de la república Rito Alejo del Río, excomandante de la Brigada 17, llamado por algunos medios como "El Pacificador del Urabá" (El Espectador, 2012), quien fue condenado por la justicia colombiana a 25 años de prisión, por todas sus actividades fuera de la ley. Estos acontecimientos, entre otros, se relacionan directamente con un territorio potencialmente rico en biodiversidad y yacimientos de minerales preciosos, motivos para la ambición por el dominio territorial de actores externos al territorio.

En breve resumen, el contexto geográfico de Quibdó (Chocó), municipio que se ubica al noroccidente de Colombia y se extiende sobre $46.530 \mathrm{~km}^{2}$. Es el único departamento de Suramérica que tiene salida al océano Pacífico y al océano Atlántico, aquí se encuentran las selvas del Darién, circundadas por los ríos Atrato y San Juan, estos ríos han sido fuente de la economía, comercio, poblamiento de esta región del país y de su identidad social. Aunque la mayor parte de la población es afrodescendiente, se encuentran comunidades indígenas como los Embera, Chamí, Embera Katío, Tule, Waunan, comunidades que se encuentran en 119 resguardos, ubicados en 26 municipios del departamento, con una población proyectada a 2011 de 54000 habitantes (Incoder, 2010) y DANE (2010). Aparte de su riqueza cultural, es un departamento rico en fauna, flora, minerales y tiene una de las tasas más alta de pluviosidad del planeta.

Allí en la ciudad de Quibdó, su capital, con una población de 112.886 habitantes, según datos del Censo 2005 (DANE, Departamento Administrativo Nacional de Estadística), su extensión territorial es de $3.693 \mathrm{~km}^{2}$. La principal actividad productiva se concentra alrededor del comercio, constituyéndose en eje de intercambio comercial y administrativo con los municipios de alto y medio Atrato. Aquí se ubica la sede principal de la Universidad Tecnológica del Chocó que desarrolla sus actividades principalmente en las instalaciones ubicadas en la Ciudadela Universitaria del barrio Nicolás Medrano, donde funciona con la modalidad de educación presencial y a distancia. Con la modalidad a distancia cubre actualmente regiones como: San Juan (Tadó, Condoto e Istmina), la Costa Pacífica (Bahía Solano, Nuquí y Pizarro), la Costa Atlántica (Acandí), y otros municipios del Departamento, como Bagadó, Lloró, Bojayá y de Antioquia (Carepa, el Bagre, Arboletes, San Pedro de Urabá), programas académicos que oferta esta universidad: Ingeniería Agroforestal, Ingeniería Ambiental e Ingeniería Civil, Tecnólogo en Gestión Minero Ambiental de los Metales Preciosos. 
Es importante resaltar que en épocas prehispánicas, los ríos que se despliegan en todo el territorio chocoano brindaban a los pobladores la posibilidad de navegarlos, sirviéndoles para penetrar y ocupar todo el espacio geográfico, logrando construir comunidades con una profunda identidad territorial, como lo afirma Mosquera (2012):

Los KUNA, fueron el pueblo indígena más importante que habitó Quibdó, estaban por todo el Golfo de Uraba y el bajo Atrato, en el mismo territorio chocoano habitaban CITARAES que se ubicaban en el Alto Atrato, estos últimos fueron los que les dieron más dolores de cabeza a los conquistadores españoles, como grupo indígena, pertenecían a la gran Tribu Caribe que sobresalió por ser muy aguerrida, también eran de baja estatura, de color cobrizo, espalda ancha, ojos negros y redondos y frente angosta. Construían sus casas a las orillas de los ríos para movilizarse en sus piraguas y facilitar la pesca; otros grupos indígenas, fueron los NOANAMAES, ubicados en la cuenca del río San Juan y los BAUDOES que habitaron en el río Baudó y Costa Pacífica (p. 34).

Con diferencias profundas, se puede evidenciar buceando en su historia que antes del descubrimiento de América en 1492, existía una gran tensión entre los citarraes y los chocoes, esta disputa territorial fue aprovechada por los conquistadores, entre ellos Martín Bueno de Sancho de Cartago, llamado El vendedor de los pijaos, quien penetró al Chocó en 1628 con 11 soldados españoles y algunos indios amigos (Mosquera, 2012). Los chocamas recibieron con júbilo a los expedicionarios, por la guerra que tenían contra los citaraes. No obstante, continuaban las tensiones, ahora entre los españoles y los indígenas y la beligerancia de estos últimos, Mosquera (1866), hace que la corona pida ayuda a los franciscanos, quienes tuvieron algún relativo éxito frente a los citaraes logrando con fray Matías Abad en 1648, quien consiguió pacificar a las comunidades indígenas, mediante el proceso de evangelización con la llamada primera doctrina en San Francisco de Atrato.

\section{Fundación de Quibdó}

No es claro el momento histórico en que fue fundada Quibdó, una primera versión indica que el padre Francisco Onetti en conferencia de 1924 dice rotundamente que los misioneros jesuitas fundaron en 1620 lo que es hoy es la capital del Chocó: Quibdó. Una segunda versión indica que el 8 de julio de 1654 Quibdó fue fundada por el teniente y alcalde Manuel Cañizales; vale la pena resaltar como una tercera versión, que antes de esta fecha el capitán Joaquín Berro fundó en el sitio Pueblo Viejo en 1643, otra población con el nombre de Citará que fue incendiada por los indios citaraes. Por su parte Manuel Cañizales en busca de minas de oro, se dirigió al sitio donde hizo su fundación, y construyó la primera casa en tierra de los caciques Guasebá (Mosquera, 2012, p. 35).

La conquista espiritual ensayada por los misioneros franciscanos a partir de $1648^{5}$ con fray Matías Abad fue la que hizo posible los asentamientos (Alcaldía de

5 Como un hecho de importancia es la "actividad misionera de Fray Matías Abad, quien en 1648 evangelizó a nuestros indómitos aborígenes y con ellos sembró en Este terruño el amor, devoción y consagración al Seráfico 
Quibdó, s.f.). A la ciudad se le han dado muchos nombres, entre ellos: Citará, San Francisco de Quibdó, La Villa de Asís, Perla Morena del Pacífico, Ciudad de Madera (Mosquera, 2012, p. 37), 1690 ya existía Citará (Quibdó) no únicamente como campo minero sino como sede o centro administrativo y del tesoro real.

\section{Distribución de la población en el espacio físico de Quibdó entre 1700 y 1780}

Los españoles o blancos habitan el centro del poblado, las comunidades indígenas viven al oriente del río Atrato (zona pantanosa), mientras tanto, las comunidades negras viven en los extremos norte y sur del poblado que dispone de templo, plaza y parque. Dada la importancia que tiene la religión católica, en 1704 San Francisco de Quibdó toma el carácter de Parroquia, en 1724 el obispo de Popayán crea la parroquia de Santa Bárbara del Citará en Quibdó. En este mismo año, ya estaba arraigada, sobre todo entre los indígenas, la devoción a San Francisco de Asís, cuya fiesta celebraban después de las cosechas, concentrándose en San Francisco de Quibdó cada 4 de octubre para festejar al Patrono,

las fiestas de "San Pacho", es un acontecimiento que está cimentado en las cosmovisiones de las comunidades como punto de encuentro de diversidad cultural, inicia el 20 de septiembre y finalizan el 4 de octubre, evento que une a toda la comunidad, especialmente a los barrios franciscanos que salen al carnaval con carrosas y comparsas (E., 2014).

\section{Después de la Independencia de la corona española}

El 2 de febrero de 1813 apropiándose del territorio y enfrentando sus miedos y pasiones, se levanta el pueblo de Quibdó en la plazoleta del templo de San Francisco, frente a los atropellos de España, replicando lo acontecido en el resto de provincias. No obstante, el 6 de mayo de 1816, el Atrato es invadido o sitiado por los españoles en la Época del Terror con Julián Bayer, se toma a Quibdó y persigue a los patriotas y ocho años después, ya habiéndose independizado de la corona española, con la Ley del 25 de junio de 1824 el departamento de Cauca se divide en cuatro provincias, y la provincia de Chocó con capital en Quibdó contiene dos cantones, el primero acentado en el Atrato con su cabecera en Quibdó, y el segundo San Juan, con cabecera en Nóvita. Es importante destacar que durante el proceso de independencia de Colombia, hay una decisión administrativa que demarcaría el rumbo del Pacífico colombiano, especialmente el Chocó. El vicepresidente Zea (1822) hace una transacción con la prestamista inglesa Herring, Graham \& Powles, quienes otorgan un préstamo de 2.000.000 de libras esterlinas y como prenda de garantía en lo que se denominó Emprestito Zea, se otorga la explotación de los recursos minerales del Chocó. Con este acontecimiento comienzan a otorgarse certificaciones de posesión de minas a perpetuidad, amparados en la Ley 292 de 1875. A pesar de la Guerra de

de Asís (El año es verídico y lo sustenta fray Luis Carlos Mantilla, en su obra: Los franciscanos en Colombia (t. 1)" citado por (Mosquera, 2012, p. 37). 
los Mil Días, acontecida en el país y que afectó al municipio con asesinatos, hambre y desplazamiento, un hito relevante para la comunidad chocoana es la llegada en 1894 de la empresa Chocó Pacífico, destinada a la explotación de oro y platino, dirigida por Henry Grange, esta compañía (Leal, 2009) a través de una serie de artimañas y aprovechando los vacíos en la legislación se hizo a 113 minas y obtuvo licencia para dragar los ríos, lo que le permitió entre 1916 y 1930 explotar las riquezas cuando los precios internacionales del platino estaban en lo más alto.

[...] esta compañía utilizó tres dragas: una comprada por la ACDC y dos más que llegaron en 1920 y en 1923. Durante el auge exportó el mayor porcentaje de platino colombiano y en 1925 la empresa Chocó Pacífico terminó de dragar las minas René y Lincoln, y al año siguiente pasó a explotar los últimos cinco kilómetros dragables de este río. Como no tenía títulos para esa sección, finalmente utilizó la concesión y comenzó a pagar regalías al Estado. Pero como lo afirma Leal (2009) durante nueve años, entre 1916 y 1925, la compañía no pagó un centavo al Gobierno colombiano [...] (Leal, 2009).

Estos acontecimientos quedaron en la memoria del pueblo y ha pasado de generación en generación, como se puede apreciar en las entrevistas, cuando afirman:

Vinieron los gringos y trabajaron todo y se llevaron toneladas de oro [...] a los políticos les interesaban que les dieran su plata y su oro y los gringos les tapaban la boca, y ellos explotaban la tierra, construyeron todo eso y dejaron luego todo tirado [...] (E., 2014).

Afirmación que evidencia para la época la impotencia del pueblo chocoano frente a las políticas institucionales que permiten a los actores (multinacionales) explotar los recursos minerales y naturales a su voluntad y casi sin ninguna restricción.

Desde el final del siglo XIX (Mosquera, 2012) la población sufre épocas de caos, estancamiento, intrigas políticas, agresiones, fracasos administrativos y poco desarrollo material, pero a comienzos del siglo XX, se establecen los sistemas de concesiones para la explotación de metales preciosos en los causes y vegas de los ríos navegables, reaparecen las grandes multinacionales y con ello incrementa la contaminación de los ríos, entre otras causas por el uso de mercurio. Casi en el mismo periodo llegan los frailes capuchinos, se resalta la importancia de la formación de los habitantes, y en 1905 los hermanos marixtas y los frailes capuchinos crean el primer colegio “Colegio Público, Instituto Pedagógico, Escuela Superior” y, finalmente, en 1915 el "Colegio Carrasquilla". En 1908 la Santa Sede asigna a los claretianos la administración de la iglesia local, huérfana desde la expulsión de los frailes capuchinos. Siguiendo esta línea en la formación de los habitantes, el 19 de marzo de 1912 llegan las hermanas dominicas de la Presentación, quienes realizaron una altruista labor en todo el Chocó y crearon el Colegio de la Presentación. Lo que permite observar la importancia de los estamentos eclesiásticos en la formación de los habitantes del Chocó. 
El año de 1919 es quizás el año más importante para el desarrollo de la región, se crea la Empresa Electrificadora del Chocó, hoy en día llamada Empresa Distribuidora del Pacífico S.A. E.S.P. (DISPAC):

[...] Empresa de Servicios Públicos Mixta que tiene por objeto la distribución y comercialización de energía eléctrica en 15 municipios del Departamento del Chocó. En diciembre del año 2001, el Gobierno Nacional constituyó la Empresa con el fin de solucionar la problemática que tenía el Departamento, con la prestación del servicio de energía por parte de la Electrificadora del Chocó. El modelo de gestión adoptado estableció que la propiedad de la Empresa quedará en manos de la Nación y que la administración y operación de la misma estuviera a cargo de una entidad privada (DISPAC, s.f.).

En la actualidad abastece de energía eléctrica a todo el municipio lo que permite a la comunidad compartir en noches con temperaturas agradables, juegos de mesa, escuchar música, mientras los niños juegan en las calles y otros adultos dialogan rememorando el pasado (E., 2014).

\section{Entre 1922 y 1940}

El 5 de agosto de 1923 acuatiza en el Atrato el primer hidroavión, lo que permite que la comunidad de Quibdó quede vinculada en la llamada Ruta Bolivariana, que se entrelazaba con Panamá, Barranquilla y Ecuador, mejorando las comunicaciones y con ello el comercio por el río Atrato. Como hecho relevante y complemento al acceso a la educación y futuro de las comunidades, en 1925 se popularizó la educación en Quibdó con la creación de la Escuela Modelo en la carrera 2a , allí asistían niños de diversas condiciones sociales.

Cuando el desarrollo estaba sobre ruedas y sin que la población quibdoseña se lo esperara, en 1926, el 12 de enero ocurre un grave incendio en Tres Esquinas; las llamas destruyen las casas en madera de los asentamientos urbanos Mario Ferrer, Delfino Díaz, Desiderio Díaz y Juan Garcés. No obstante a la devastación empieza la reconstrucción y aprovechando esto, en 1929, más específicamente el 20 de julio, es inaugurado para bien de la comunidad el Hospital San Francisco de Asís. Es importante resaltar que entre 1920 y 1935 como bien lo expresa (Mosquera, 2012, pp. 47-51) la economía de la región estaba basada en:

[...] la explotación de sus recursos mineros, pesqueros, fauna y flora, forestales, agropecuarios en mínima escala [...] con grandes obras y fábricas de jabones, gaseosas, licores, baldosines, hielo, bujías esteáricas, aserríos, velas, entre otros, calles pavimentadas en que corrieron varios automóviles, de los primeros que llegaron a Colombia. Los principales sectores barriales hacia 1933 eran: Yesquita, Yesca Grande, Alameda Reyes, Belén de Judá, Barrio Central y Barrio Norte (esto según monografía inédita de Quibdó por Andrés Fernando Villa) (pp. 47-51). 
Aspectos que llevan al establecimiento de la Cámara de Comercio de Quibdó creada por el Decreto 662 del 8 de abril de 1935 con jurisdicción en todos los municipios del departamento del Chocó, excepto en el municipio de San José del Palmar, el cual es cubierto por la Cámara de Comercio de Cartago.

Como complemento a la formación básica de los habitantes, en 1934 y en 1936 comienzan labores el Instituto Intendencial para Damas y la Escuela Normal para Varones, con palabras del doctor Diego Luis Córdoba "Yo les aseguro a las madres chocoanas que pronto sus hijas cambiarán el delantal de sirvientas por diplomas de maestra [...] y los varones también cambiarán la pala y el azadón por el diploma de maestros" (Mosquera, 2012, p. 63).

Años más tarde y pensando en la capacidad de desarrollo y empleabilidad se crea el SENA, específicamente

durante el gobierno de la Junta Militar, posterior a la renuncia del general Gustavo Rojas Pinilla, mediante el Decreto-Ley 118, del 21 de junio de 1957. Su función, definida en el Decreto 164 del 6 de agosto de 1957, fue brindar formación profesional a trabajadores, jóvenes y adultos de la industria, el comercio, el campo, la minería y la ganadería (SENA, s.f.).

Con el objetivo de buscar y proporcionar la formación técnica y complementaria de los empleados, lo que permitió el desarrollo de trabajos en la artesanía y el trabajo con filigranas en oro y ebanistería.

Como un fantasma que aparece de improvisto, el incendio del 26 de octubre de 1966 arrasó con todo el sector comercial y zonas residenciales, posterior a este incendio y a iniciativas gubernamentales de reconstrucción y tipificación del territorio, se crea Codechoco y en 1967 se edifica una nueva plaza de mercado, después del incendio de 1966 ubicada en el sector sur occidental de la ciudad a orillas del río Atrato. En 1972 inicia un nuevo período, con el relleno de terrenos, las personas dejan de construir casas de madera para hacerlas de concreto, mejoran las edificaciones públicas y gubernamentales y en 1972, Quibdó llega a su culmen en la educación con la puesta en marcha del Instituto Universitario Diego Luis Córdoba, siguió laborando con el nombre de Universidad Tecnológica del Choco $^{6}$ (UTCH). El 7 de marzo de 1972 con labores académicas en jornada nocturna en las aulas del Colegio Carrasquilla. Lo que marca nuevas condiciones de vida de los pobladores.

En términos del ordenamiento territorial de Quibdó, entre 1970 y 1980 se alargan sus vías principales, mayoría de las casas cambian su estructura pasando de madera a concreto, sus calles son pavimentadas, tienen todos los servicios de aseo. Pero

6 La Universidad Tecnológica del Chocó "Diego Luis Córdoba" es una institución pública de carácter académico del orden nacional, con personería jurídica, autonomía administrativa y patrimonio independiente, adscrita al Ministerio de Educación Nacional, creada mediante la Ley 38 de 1968 y Ley 7 de 1975 y reconocida como ente universitario autónomo por la Resolución 3274 de 1993, emanada del Ministerio de Educación Nacional. La sede principal es la ciudad de Quibdó, con programas académicos que cubren las regiones del San Juan (Tadó e Istmina) y la costa Pacífica (Bahía Solano). Extractado de: http://portal.utch.edu.co/es/acerca-de-utch/ informacion-general.html 
justo cuando la comunidad tiene un sentimiento de prosperidad, inicia un proceso de oscurantismo provocado por una nueva etapa en la violencia del país.

\section{Violencia en el Chocó desde 1980 hasta nuestros días}

La bonanza cocalera de la década de los ochenta y parte de los noventa, convirtió al departamento del Chocó en una de las rutas predilectas del narcotráfico dada la importancia que tiene este departamento por su salida a los mares Atlántico y Pacífico y su imponente vegetación de bosque tropical húmedo, espacio utilizado por los narcotraficantes como refugio y a la vez para la creación de laboratorios para el procesamiento de la cocaína. En este mismo período de tiempo ingresan al departamento del Chocó las fuerzas armadas revolucionarias de Colombia (FARC) y con la incursión en 1997 de los grupos paramilitares, empieza una guerra indiscriminada por el dominio del territorio. Los grupos armados ilegales en sus iniciativas de control y dominio, crean estrategias de exterminio a la población civil con la llegada entre 1995 y 1996 de las autodefensas del Urabá antioqueño, dejan por doquier todo tipo de actos violentos, y con ello desplazamientos forzados de la población, lo que ahumenta el asentamiento en las ciudades principales entre ellas Quibdó:

[...] todos estábamos bien en los pueblos, lastimosamente por el problema del conflicto armado la gente empezó a desplazarse [...] si, empezó a desplazarse. Hay ciertas zonas del departamento que la gente básicamente no puede convivir bien. Otra cosa que han hecho el conflicto armado, otro factor negativo son los cultivos ilícitos, en algunos sectores, la gente empezó a cambiar el imaginario, la gente ya no cultiva si no coca; dejó de pescar en las costas y al meterse en el negocio, y empezamos a ser un poco más dependientes de afuera, empezó a encarecer todo, y han cambiado la dinámica del pueblo, el concepto del territorio se ha visto contaminado por fuerzas externas, conflicto armado (E., 2014).

Entre 1990 y 2000 a causa de la violencia hay un aumento desorganizado en el aspecto territorial de la ciudad, los “[...] asentamientos son espontáneos, sin servicios públicos y expuestos a deslizamientos en las lomas o colinas y a inundaciones de las quebradas. Se presenta el fenómeno de los habitantes desplazados provenientes del Medio Atrato" (Mosquera, 2012, p. 47), luego, con la Ley 99 de 1993 se crea el Instituto de Investigaciones Ambientales del Pacífico (I.I.A.P.); sin embargo, inicia sus funciones como institución a partir de 1996, cuya misión busca "actuar como instrumento de coordinación y apoyo al fortalecimiento de la capacidad de investigación de la región y de sus actores sociales e institucionales" (I.I.A.P). Este proyecto es bienvenido para las comunidades que se preocupan por realizar trabajos e investigaciones que impacten en la sustentabilidad de las "ciénagas que son importantes por su biodiversidad, muchas aves, muchas especies, caimanes, tortugas, al producir minería en un caño de los que alimentan la ciénaga, las arenas contaminaban, unas especies se mueren otras se salen" (E., 2014). 
En 1991, el país pasa por un momento histórico que repercutirá en el futuro de los territorios de las comunidades ancestrales. Se realiza una Constituyente que busca replantear una constitución nacional que sea más justa y permita la inclusión en la diferencia. Con base en la Carta Magna, en 1998 el Gobierno Nacional expide el Decreto 1320 llamado consulta previa, que es el reconocimiento al derecho fundamental que tienen los pueblos ancestrales a sus territorios, reconocido por las Naciones Unidas mediante el Convenio OIT 1691 de 1989 e incorporado en la legislación nacional por la Ley 21 de 1991 que como lo aseguran las comunidades "Busca salvaguardar las personas, sus bienes, y al medio ambiente, protegiendo nuestros valores y creencias, patrimonio de nuestros futuros pobladores" (E., 2014). Así, para las comunidades, la normatividad legal se convierte en el elemento de poder que es utilizado como defensa ante las arremetidas de las multinacionales que buscan explotar los recursos naturales y minerales, en este sentido manifiestan:

[...] el Gobierno nos considera los pobrecitos, allá, pero tenemos un as bajo la manga, nosotros tenemos el suelo titulado, la multinacional tiene que negociar, el código está mal negociado, toman todo con la fuerza, metiendo a la policía, no pueden venir a decir por ejemplo a un barequero que tiene que estar afiliado a una organización, esa propuesta del código debe ser la consulta previa, es un mandato de la OIT, con su convenio 169, ratificada en Colombia por la Ley 21, que específica que debe ser previamente consultado y de buena fe [....] nosotros como afros y como indígenas, tenemos una connotación muy diferente con una legislación que nos ampara, para hacer uso, de los recursos naturales [....] La primera norma nuestra es la Ley 70, sus decretos reglamentarios, la Ley 99 con la creación del Sistema Nacional Ambiental, el Decreto 1320 de consulta previa, a pesar de la Sentencia unificada 039 del 97 que da pie para que la consulta se aplique, la Constitución del 91 y otros decretos [...], la Ley 70, todo lo que hagamos debe girar en torno al desarrollo de las comunidades y al bienestar de estas, es necesario evaluar cómo esto aporta al desarrollo sostenible (E., 2014).

Este reconocimiento de las comunidades y la falta de una política clara del Estado, frente a la arremetida de grupos ilegales armados, causa tensiones y es así como después del 2006 inicia un nuevo período el posparamilitarismo, aparecen grupos emergentes configurando nuevos focos que inciden en el aumento de violencia, desplazamiento forzado, secuestros y asesinatos, la mayoría desplazados por el conflicto armado, como se puede observar en los informes del Programa presidencial de derechos humanos y derecho internacional humanitario de la Presidencia de la República, donde se registraron los acontecimientos violentos entre el 2003 y el 2008, con un total de:

[...] 311 personas asesinadas, 26 desplazamientos masivos en los cuales 12.380 personas salieron de sus lugares habituales de vivienda. En el año 2004 se desplazaron 555 familias de Bojayá, 256 de Istmina, 62 de Condoto, 15 de Alto Baudó y 14 de Quibdó. En 2005, lo hicieron 481 familias de Bojayá y 167 de Bajo Baudó. En 2006, fueron 270 familias de Istmina y Condoto. En 2007, fueron 217 familias de Sipí, 147 de Quibdó, 136 de Condoto y 20 del Litoral de 
San Juan. Por último, en el año 2008 se desplazaron 331 hogares de Istmina, 217 de Alto Baudó, 95 de Bajo Baudó y 23 de Litoral de San Juan (Observatorio del Programa Presidencial de DH y DIH, s.f.: 41)

En el mismo comunicado muestran cómo la violencia es causada por el accionar de los grupos guerrilleros y paramilitares, quienes con su locura y ambición de poder, masacran indiscriminadamente a la sociedad civil. Sociedad que tiene información de los acontecimientos vividos gracias al primer canal de televisión regional Tele Quibdó (creado en 1988) que permite a la comunidad estar enterada casi en tiempo real de los acontecimientos en el país y el resto del mundo, por tanto queda en el imaginario de la población que:

[...] los problemas que genera la violencia son pobreza descomposición social, un desplazamiento masivo, con deterioro de las comunidades, engrosamiento del área delincuencial. El Gobierno no hace nada para que eso se solucione; la violencia no es solo el grupo al margen de la ley, sino que es también del Gobierno, supuestamente el Gobierno conoce todo, el Gobierno patrocina la violencia, en pocas palabras un caos social (E., 2014).

Pero no todo es violencia en el departamento, gracias a la tenacidad y diversidad cultural de sus habitantes, logran que el Gobierno emita la Ley 993 de 2005, que visibiliza y reconoce su diversidad cultural representada en las fiestas de San Pacho, declarada Patrimonio Cultural Inmaterial de la Nación, y mediante la Ley citada, se establece la Fundación Fiesta Franciscana de Quibdó; goza de estatutos propios y personería jurídica, (0421 del 11 mayo de 1981); esta fiesta permite manifestar los sentimientos más profundos de sus habitantes "para nosotros los habitantes de Quibdó, las fiestas de San Pacho nos permiten no olvidar nuestro pasado, recatar nuestras costumbre e identidad y visibilizarnos ante el mundo" (E., 2014) y el 20 de septiembre del 2011 el Gobierno Nacional, mediante Resolución No. 1895 incluyó la fiesta patronal San Pacho (Colombia Travel, s.f.). en el listado de las fiestas representativas de la nación y "nuestra festividad, sea declarada Patrimonio Cultural Inmaterial de la Humanidad, por la UNESCO [...] nosotros los pobladores ancestrales recordamos todo nuestro pasado, nuestra cultura, nuestra relación con Dios, nuestra resistencia a la esclavitud y nuestra dignidad como seres humanos" (E., 2014).

Volviendo con el territorio vivido y con la minería, la sociedad de cabildos indígenas del Chocó (OREWA), realiza demandas frente a la intervención de la megaminería en el Chocó, resaltando que hasta el 2010 se alcanzaron a entregar 157 títulos mineros por 30 años, y prorrogables por otros 60 años más, para la explotación minera en el departamento del Chocó; en una proporción muy importante fueron entregados a grandes empresas mineras multinacionales, en particular a la Anglo Gold Ashanti, resaltando el impacto de estas prácticas frente al despojo territorial de los pueblos indígenas del Chocó la minería va contaminando al agua y también a los peces, antiguamente había gran cantidad de peces, los peces ahora en la minería por estar trabajando con el mercurio han acabo con muchos peces el azogue de la minería con Mercurio" (E., 2014). 
Es evidente la nostalgia que tienen las comunidades cuando evocan su vida y relación con el territorio en tiempos pasados de continua interacción y respeto con la madre tierra, esto entra en contravía con la fuerte presencia de los grupos armados ilegales, lo que ha conllevado el asesinato de los líderes comuneros y autoridades indígenas del territorio chocoano.

\section{Conclusión}

Articular el sentir de la población quibdoseña, sus hitos territoriales y las dimensiones para análisis del territorio (Bustos, 2015), se puede inferir que en la dimensión construcción social se encuentra una relación directa con las posturas de investigadores, como: Quijada (2000), Bozzano (2009, 2012), Fals (2000) y Santos (2000) que muestran las múltiples interacciones dinámicas que tienen los actores en el territorio, donde la relación comunidad, educación, cultura, desarrollo y usurpación configuran un escenario complejo en la vida de los habitantes, que desde una postura crítica ven en las decisiones políticas una falta de control y vigilancia del Estado. Específicamente frente a la explotación minera, que de acuerdo con Olivero, Caballero y Negrete (2011) generan un impacto negativo en los sistemas de flora y fauna, lo que deja unas pocas monedas en las arcas del Estado y mucha pobreza y miseria a la población, despropiándola de los elementos propios para desarrollarse socioculturalmente en el territorio y que ve en la normatividad (Constitución Política de 1991, Ley 70 y el Decreto 1745) un respaldo a la posesión y defensa del territorio ancestral.

Otro aspecto que vale la pena resaltar, está relacionado con la dimensión ambiental, donde las comunidades expresan la necesidad de un desarrollo sustentable del territorio, concepción que comparten a nivel latinoamericano (Leff, 2006; Irastorza, 2006; Marris, 2008) quienes invitan a pensar en el desarrollo de una buena calidad de vida, en la que se respeten los valores y conocimientos culturales y al mismo tiempo se promueva la conservación ambiental para que gocen de esto las generaciones futuras. Por último, en la dimensión Espacio físico, la comunidad reconoce los límites y el espacio habitado, en tanto que ambos demarcan diferencias y tensiones entre los actores que convergen en el territorio chocoano, resaltando la importancia de los títulos colectivos como la posibilidad de tenencia del territorio habitado ancestralmente.

\section{Referencias}

Alcaldía de Quibdó Chocó. (s.f.). Recuperado de https://www.quibdo-choco.gov.co/ Paginas/Inicio.aspx.

Bozzano, H. (2009). Territorios posibles. Procesos, lugares y actores. Buenos Aires: Lumiere. 
Bustos, E. (2015a). Tendencias investigativas en territorio y su importancia en la formación de profesionales. Revista Lebret, 7, 261-279. Bucaramanga, Colombia: Universidad Santo Tomás.

Cámara Colombiana del Chocó. (s.f.). Recuperado de: http://www.camarachoco.org. co/quienes-somos/historia.

Colombia Travel. (s.f.). Recuperado de http://www.colombia.travel/es/turistainternacional/actividad/historia-y-tradicion/ferias-y-fiestas/septiembre/fiestasde-san-pacho-en-quibdo.

Departamento Nacional de Estadística DANE. (2010). Datos sobre proyecciones de población indígena en resguardos a 30 de junio de 2010.

Departamento Nacional de Planeación. (2011). Apuntes sobre la consulta previa con grupos étnicos. Documento de trabajo interno. Recuperado de https://www.dnp. gov.co/LinkClick.aspx?fileticket=Grq2aX7b9gU\%3D\&tabid=273.

Empresa Distribuidora de Servicios (DISPAC). (s.f.). Recuperado de http://dispac. com.co/nuestra-empresa/resena-historica/

Instituto Colombiano de Desarrollo Rural INCODER. (2013). Implementación del programa de legalización de tierras y fomento al desarrollo rural para comunidades indígenas a nivel nacional. Bogotá.

Irastorza, P. (2006). Integración de la ecología del paisaje en la planificación territorial. Aplicación a la comunidad de Madrid. (Tesis doctoral). Universidad Politécnica de Madrid.

Leal. C. (2009). La compañía minera Choco Pacífico y el auge del platino en Colombia, 1897-1930. Historia Crítica. Edición Especial, pp. 150-164.

Leff, E. (2006). Aventuras de la epistemología Ambiental. México D. F.: Siglo XXI Editores.

Maris, S. (2008). Territorio y sustentabilidad. El "caldenal” en la lógica actual del capitalismo, Revista Iberoamericana de Economía Ecológica. 9: 61-73.

Molina, A., Mosquera, C., Utges, G., Mojica, L., Cifuentes, M., Reyes, D., Martínez, A., y Pedreros, R. (2014). Concepciones de los profesores sobre el fenómeno de la diversidad cultural y sus implicaciones en la enseñanza de las ciencias (p. 233). Bogotá: Universidad Distrital Francisco José de Caldas.

Mosquera. (1866). Geografía general política, física y especial de los Estados Unidos de Colombia. Londres: Imprenta Inglesa y Extranjera de H. Panzer.

Mosquera. O. (2012). Semblanzas de Quibdó. La Alegre Villa de Asís. Oficina de Comunicaciones IIAP. 
Observatorio del Programa Presidencial de DH y DIH. (s.f.). Diagnóstico de la situación de los municipios habitados por las comunidades afrocolombianas priorizadas por la Honorable Corte Constitucional en el departamento de Chocó. Recuperado de http://historico.derechoshumanos.gov.co/Observatorio/ documents/2010/DiagnosticoAfro/Choco.pdf

Olivero, V., Caballero, G., \& Negrete, M. (2011). Relationship between localization of gold mining areas and hair mercury levels in people from Bolivar, north of Colombia. Biological Trace Element Research, 144(3), 118-132.

Quijada, M. (2000). Indígenas: Violencia, tierras y ciudadanía. En Quijada, M. y otros. Homogeneidad y nación. Con un estudio de caso: Argentina, Siglos XIX y $X X$. Madrid: Csic.

Santos, M. (2000). La naturaleza del espacio, técnica y tiempo. Razón y emoción. Barcelona. España: Ariel.

Servicio Nacional de Aprendizaje (SENA). (s.f.). Recuperado de http://www.sena. edu.co/acerca-del-sena/quienes-somos/Paginas/Historia-Vision-Mision-Valoresy-Simbolos.aspx.

Universidad Tecnológica del Chocó. (S.F.). Recuperado de http://portal.utch.edu.co/ es/acerca-de-utch/informacion-general.html. 